\title{
Development of Lead-Free Nanowire Composites for Energy Storage Applications
}

\author{
Miguel Mendoza, Md Ashiqur Rahaman Khan, Mohammad Arif Ishtiaque Shuvo, \\ Alberto Guerrero, and Yirong Lin
}

Department of Mechanical Engineering, The University of Texas at El Paso, El Paso, TX 79968, USA

Correspondence should be addressed to Yirong Lin, ylin3@utep.edu

Received 31 August 2012; Accepted 25 September 2012

Academic Editors: J. Blázquez and A. A. Ismail

Copyright (C) 2012 Miguel Mendoza et al. This is an open access article distributed under the Creative Commons Attribution License, which permits unrestricted use, distribution, and reproduction in any medium, provided the original work is properly cited.

\begin{abstract}
There is an increasing demand to improve the energy density of dielectric capacitors for satisfying the next generation material systems. One effective approach is to embed high dielectric constant inclusions such as lead zirconia titanate in polymer matrix. However, with the increasing concerns on environmental safety and biocompatibility, the need to expel lead ( $\mathrm{Pb}$ ) from modern electronics has been receiving more attention. Using high aspect ratio dielectric inclusions such as nanowires could lead to further enhancement of energy density. Therefore, this paper focuses on the development of a lead-free nanowire reinforced polymer matrix capacitor for energy storage application. Lead-free sodium niobate nanowires $\left(\mathrm{NaNbO}_{3}\right)$ were synthesized using hydrothermal method, followed by mixing them with polyvinylidene fluoride (PVDF) matrix using a solution-casting method for nanocomposites fabrication. Capacitance and breakdown strength of the samples were measured to determine the energy density. The energy density of $\mathrm{NaNbO}_{3} / \mathrm{PVDF}$ composites was also compared with that of lead-containing $(\mathrm{PbTiO} / \mathrm{PVDF})$ nanocomposites and previously developed $\mathrm{Pb}\left(\mathrm{Zr}_{0.2} \mathrm{Ti}_{0.8}\right) \mathrm{O}_{3} / \mathrm{PVDF}$ composites to show the feasibility of replacing lead-containing materials. The energy density of $\mathrm{NaNbO}_{3} / \mathrm{PVDF}$ capacitor is comparable to those of lead-containing ones, indicating the possibility of expelling lead from high-energy density dielectric capacitors.
\end{abstract}

\section{Introduction}

Electrical energy storage plays an important role in modern electronic devices such as stationary power systems, mobile devices, and pulse power applications [1]. The most common devices used for storing electrical energy are batteries and capacitors. Compared to batteries, capacitors typically have lower power density but can be charged/discharged very quickly and has a significantly higher range of operating voltage [2]. Currently, there are many types of capacitors in use, such as dielectric capacitors, electrolytic capacitors, and electric double layer capacitors (EDLC or supercapacitors) [3]. Among these, the dielectric capacitor is still the most widely used because of its low cost, easy processing capability, low dielectric loss, high operation voltage, and reliability. Within dielectric capacitors group, there are two main types of capacitors: polymer based and ceramic based [1].
Polymer-based capacitors are more widely used because of their high breakdown strength, lightweight, and easy processing capability [1]. To date, dielectric polymer film capacitors have been used for power electronics, power conditioning, and for pulse power applications such as motors, lighting, and portable defibrillators [4]. Dielectric film capacitors are commonly used in pulse forming network (PFN) for conversion of available electrical energy into the necessary short and fast pulses of energy in the megajoule range [5]. Typical commercial polymer capacitors are shown in Figure 1(a), where the dielectric polymer film and metal electrodes are rolled together into a cylindrical shape to minimize volume, as indicated by Figure 1(b). However, polymers have low relative dielectric constants (typically $<4$ ), which limits the energy density and requires extremely high operating voltages and high breakdown strength materials to achieve high-energy density [6]. 
Ceramics are the other common material broadly used in dielectric capacitors [7]. Compared with polymers, ceramics such as $\mathrm{BaTiO}_{3}, \mathrm{~Pb}\left(\mathrm{Zr}_{x} \mathrm{Ti}_{1-x}\right) \mathrm{O}_{3}$ (PZT), and lanthanumdoped PZT (PLZT) have significantly higher relative dielectric constants. However, the breakdown strength of ceramics is typically two orders of magnitude lower than dielectric polymers [8], which results in lower energy density. Moreover, due to its fragility and brittleness, it is difficult to manufacture ceramic capacitors with desired capacity for energy storage applications [9].

Therefore, a good deal of research effort has been devoted to combine the high dielectric constant of ceramics and high breakdown strength of polymers through the composite approach. To date, many research groups increased the energy density of dielectric capacitors using a nanocomposite approach, including barium titanate $\left(\mathrm{BaTiO}_{3}\right)$ nano particles reinforced polycarbonate (PC) [10], poly(vinylidene fluoride-co-hexafluoropropylene) (PVDF-HFP) [11], modified $\mathrm{BaTiO}_{3}$ nanoparticle with PVDF $[12,13]$, titanium dioxide $\left(\mathrm{TiO}_{2}\right)$ nanoparticles with PVDF terpolymer [14], calcium copper titanate (CCT) reinforced polyimide [15], silver nanoparticle/poly (vinyl pyrrolidone) core-shell structure for high dielectric constant and low loss epoxy matrix composite [16], and PZT or PLZT powders reinforced PVDF $[1,17]$.

While the foregoing efforts have shown some improvements in energy density, the enhancement of energy density is still limited because of the use of ceramic particles or low aspect ratio nanorods [1]. Recently, Andrews et al. developed a micromechanics model to show that using higher aspect ratio nanowires instead of nanoparticle inclusions could lead to significant increase in the dielectric constant of the nanocomposites [18]. Further, Tang et al. experimentally demonstrated that the use of nanowires instead of nanoparticles could significantly increase the dielectric energy density of the nanocomposites [19]. While the use of nanowire could lead to higher energy density capacitor, most of the high dielectric constant inclusions used has lead- (Pb-) containing materials, such as PZT and PLZT, which make the resulting nanocomposites toxic and not compatible with biological applications. Expelling lead from commercial applications and materials such as solders, glass, and gasoline has been receiving extensive attention because of the concerns regarding its toxicity.

Therefore, this paper focuses on the development of a lead-free nanowire reinforced polymer capacitor with comparable dielectric properties to lead-containing capacitors. In details, sodium niobate (NNO, lead free) and lead titanate nanowires (PTO, lead containing, control samples) were first synthesized using hydrothermal methods, followed by characterization using Scanning Electron Microscopy (SEM) and X-ray diffraction (XRD) analysis. Solution casting process was then carried out to fabricate two types of nanocomposites: NNO reinforced PVDF and PTO reinforced PVDF. The dielectric constant and breakdown strength of nanocomposites with volume fractions ranging from $5 \%$ to $30 \%$ were experimentally tested to determine the energy densities of both nanocomposites. Testing results have shown that the NNO/PVDF composites have higher dielectric constants, lower dielectric loss, and comparable energy density. Therefore, this report demonstrates the feasibility of developing lead-free high-energy polymer capacitors to ultimately replace lead-containing ones.

\section{Experimental Procedure}

The fabrication process of the NNO/PVDF and PTO/PVDF nanocomposites utilized a two-step procedure; NNO and PTO nanowires were first synthesized using hydrothermal technique, respectively, as shown in Figure 2, followed by dispersing nanowires in PVDF and dimethylformamide (DMF) solution to form nanocomposites by solution casting method. Samples with volume fractions ranging from 5\% to $30 \%$ were prepared to study the nanowire volume fraction influence on energy density.

2.1. $\mathrm{NaNbO}_{3}$ Nanowires Synthesis. Sodium niobite nanowires were synthesized following a hydrothermal method developed by Jung et al. [20]. In a typical process, a $12 \mathrm{M}$ $\mathrm{NaOH}$ solution was prepared by dissolving $33.6 \mathrm{~g}$ of $\mathrm{NaOH}$ (Acros Organics, 98\%) into $70 \mathrm{~mL}$ of deionized water (DI). Subsequently, $3.5 \mathrm{~g}$ of $\mathrm{Nb}_{2} \mathrm{O}_{5}$ (Aldrich, 99.99\%) were added into the $\mathrm{NaOH}$ solution. After stirring for a period of $30 \mathrm{~min}-$ utes at room temperature, the mixture was transferred into a $160 \mathrm{~mL}$ Teflon lined stainless steel autoclave with a fill factor of $80 \%$. The autoclave was placed inside an electrical oven to undergo hydrothermal reaction at $180^{\circ} \mathrm{C}$ for $4 \mathrm{~h}$. After cooling down to room temperature, white precipitate was filtered, washed with DI water for several times, and dried at $80^{\circ} \mathrm{C}$ for $12 \mathrm{~h}$. Finally, NNO powders were annealed at $550^{\circ} \mathrm{C}$ for $4 \mathrm{~h}$ in order to obtain crystallized NNO nanowires.

2.2. PTO Nanowires Synthesis. PTO nanowires were synthesized using a hydrothermal method similar to those previously reported $[19,21]$. Titanium (IV) n-butoxide (Acros Organics, 99\%) ethanol solution $(0.09 \mathrm{M})$ was precipitated by $0.15 \mathrm{M}$ ammonia solution under vigorous magnetic stirring. The white precipitation was separated by centrifuge and subsequently washed three times with DI water. The gel was dispersed in DI water followed by an inclusion of lead (II) nitrate $\left(\mathrm{Pb}\left(\mathrm{NO}_{3}\right)_{2}\right)$ (Fischer Chemical, 99\%), potassium hydroxide $(\mathrm{KOH})$ (Acros, pellets, 85\%), and poly(vinyl alcohol) (PVA) (Aldrich chemistry, 99+\% hydrolyzed) in the form of aqueous solution. Final solution had concentrations of $0.11 \mathrm{M} \mathrm{Pb}\left(\mathrm{NO}_{3}\right)_{2}, 0.5 \mathrm{M} \mathrm{KOH}$, and $0.4 \mathrm{~g} / \mathrm{L}$ PVA. After continuous stirring for 30 minutes, solution was transferred into a $200 \mathrm{~mL}$ Teflon lined stainless steel autoclave with a fill factor of $80 \%$. The reactor was placed on a hot plate with a heating band to undergo hydrothermal reaction at $200^{\circ} \mathrm{C}$ for $4 \mathrm{~h}$ under vigorous stirring. After cooling down to room temperature, PTO powders were recovered through filtration, washed with DI water and ethanol for three times, and dried at $80^{\circ} \mathrm{C}$ for $12 \mathrm{~h}$. Finally, PTO powders were annealed at $600^{\circ} \mathrm{C}$ for $2 \mathrm{~h}$ to obtain crystallized PTO nanowires.

2.3. Nanocomposites Fabrication. PVDF (Aldrich, Mw 53,4000) and DMF (Acros, 99.8\%) were mixed at a $1: 10$ 


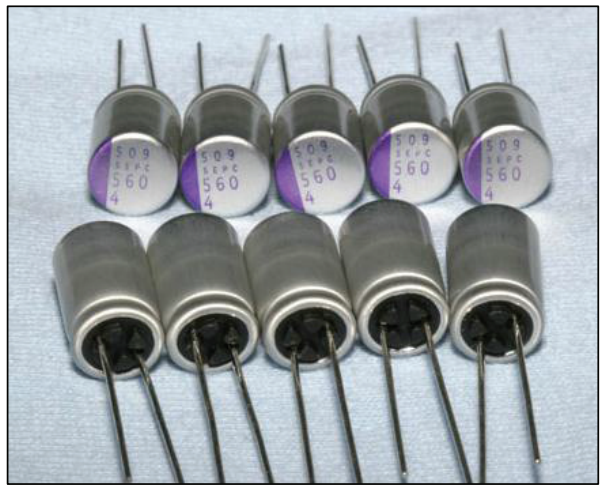

(a)

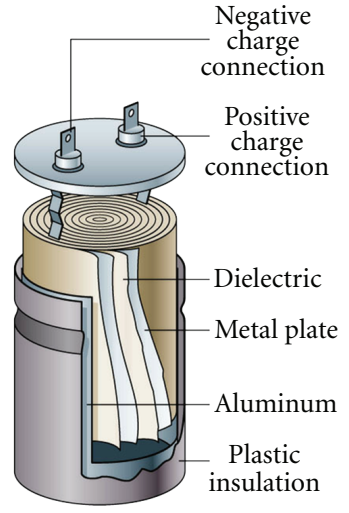

(b)

FIgURE 1: Commercial dielectric polymer film capacitors, (a) digital picture view, and (b) schematic cross-section view. Source: http://www.capacitorlab.com/.
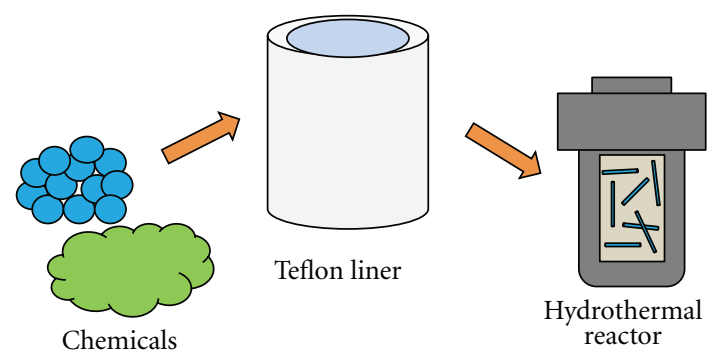

FIGURE 2: Schematic diagram for the hydrothermal process followed for growing PTO and NNO nanowires.

weight ratio and heated up at $80^{\circ} \mathrm{C}$ for 30 minutes to fully dissolve the PVDF. Nanocomposites were prepared by dispersing NNO or PTO nanowires into PVDF/DMF solution by manual stirring and horn sonication (Branson, S-450A) until a homogeneous mixture was obtained. Subsequently, solution was casted onto a PTFE film and dried at $80^{\circ} \mathrm{C}$ for $6 \mathrm{~h}$. In order to achieve a consistent thickness over the entire film, nanocomposites were hot pressed at $160^{\circ} \mathrm{C}$ for 15 minutes under a constant pressure of 1 ton (Carver, 3850). Finally, top and bottom surfaces of nanocomposites were coated with silver paint as electrodes for electrical testing. The fabrication process of the nanocomposites is schematically shown in Figure 3.

2.4. Characterization. The crystal structure of the asprepared nanowires was determined through Bruker D8 Discover XRD using $\mathrm{Cu} \mathrm{K} \alpha$ radiation. The morphology of the samples was analyzed using S-4800 SEM. Frequencydependent capacitance and dielectric loss tangent were measured using HP 4284A LCR meter within a frequency range between $1 \mathrm{kHz}$ and $1 \mathrm{MHz}$ at $1 \mathrm{Vrms}$ with a parallel equivalent circuit. The breakdown voltages were measured according to ASTM standard (ASTM D149-09) [22]. Experimental setup utilized a $30 \mathrm{kV}$ high voltage power supply (PO3HP2, Acopian) and a digital oscilloscope (DS1102E,
Rigol), both connected to a $20 \mathrm{MHz}$ function/arbitrary waveform generator (33220A, Agilent). In order to avoid arcing prior to breakdown, nanocomposites were held inside a $500 \mathrm{~mL}$ beaker filled with silicon oil. Breakdown voltage was recorded through a digital multimeter (DranTech TRMS System PRO Multimeter, Dranetz BMI) connected to the high voltage power supply.

\section{Results and Discussion}

The morphology of the synthesized PTO and NNO nanowires (NWs) is shown in Figures 4(a) and 4(b), respectively. It is shown that PTO NWs have an average diameter of $100 \mathrm{~nm}$ and an average length of $10 \mu \mathrm{m}$, while NNO NWs have an average diameter of $400 \mathrm{~nm}$ with length up to $20 \mu \mathrm{m}$. Dispersion of PTO and NNO nanowires in the PVDF polymer matrix is shown in Figures 4(c) and $4(\mathrm{~d})$, respectively. Note that the volume fractions are both $30 \%$ for nanocomposites shown in Figures $4(\mathrm{c})$ and $4(\mathrm{~d})$. As indicated from the SEM images, homogeneous dispersion of the nanowires throughout the entire sample is achieved and no voids are shown in the samples. As expected, SEM images displayed random orientation of nanowires inside the polymer matrix.

After the morphology characterization, XRD analysis of PTO and NNO nanowires was performed to characterize the crystal structure of the nanowires. The XRD peaks for the asprepared nanowires are shown in Figure 5. NNO nanowires displayed orthorhombic phase and excellent crystallinity. The calculated lattice parameters for these nanowires are $a=5.5625 \AA, b=15.5495 \AA$, and $c=5.5135 \AA$, which are similar values to the ones previously reported on literature $[23,24]$. PTO nanowires displayed tetragonal structure and lattice parameters of $a=b=3.9024 \AA$ and $c=$ $4.1583 \AA$. All PTO peaks matched with previous research efforts and indicated great crystallinity [25, 26]. For both PTO and NNO, the entire diffraction peaks match with those indicated by standard card JCPDS of PTO (no. 01074-3218) and NNO (no. 01-072-7753); no other peaks 


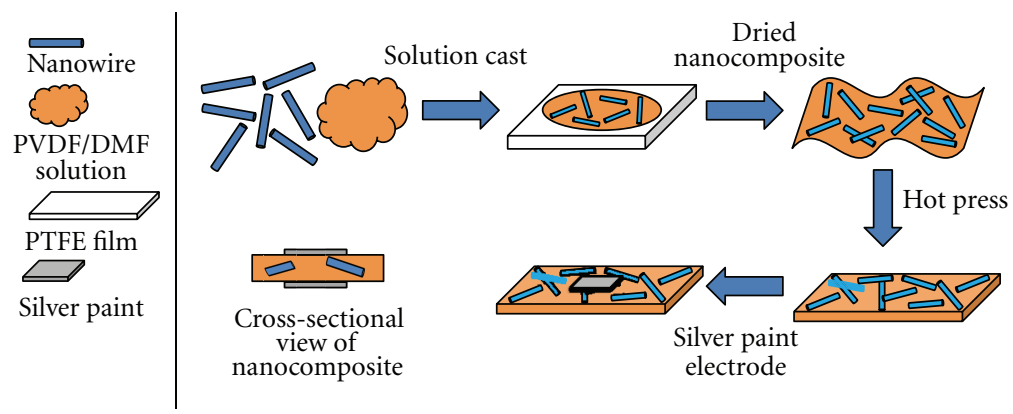

FIgURE 3: Schematic diagram of the manufacturing process of nanocomposites.

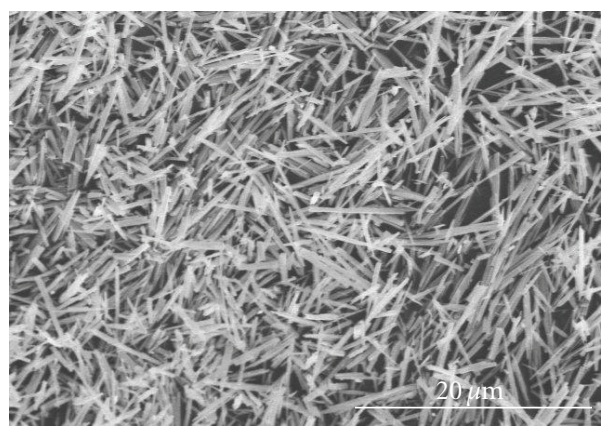

(a)

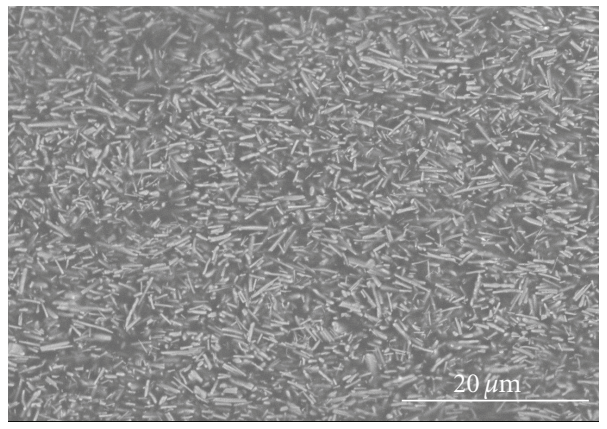

(c)

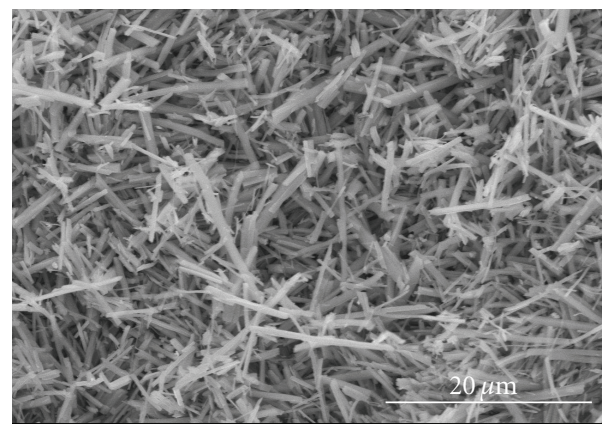

(b)

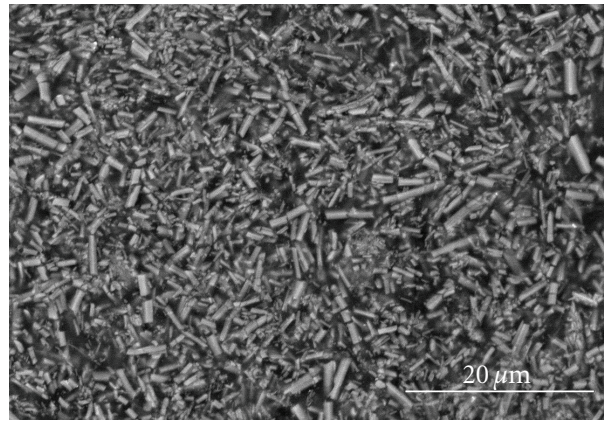

(d)

FIgure 4: SEM images of PTO and NNO NWs and nanocomposites: (a) PTO NWs, (b) NNO NWs, (c) PTO/PVDF nanocomposite, and (d) NNO/PVDF nanocomposite.

were determined, indicating that both PTO and NNO have excellent crystallinity.

Followed by the fabrication of nanocomposites, the electrodes were applied on top and bottom surfaces of the samples to form capacitors in parallel plate fashion, where the energy density is defined by [27],

$$
W=\frac{1}{2} \varepsilon_{0} \varepsilon_{r} E_{b d}^{2},
$$

where $\varepsilon_{0}$ is the permittivity of vacuum $\left(8.85 \times 10^{-12} \mathrm{~F} / \mathrm{m}\right)$, $\varepsilon_{r}$ is the relative dielectric constant, $E_{b d}=V_{b d} / t$ is the breakdown strength of the material, $V_{b d}$ is the breakdown voltage, and $t$ is the thickness of the capacitor. Therefore, in order to determine the energy density of the nanocomposites, the dielectric constant and breakdown strength must be determined. The dielectric constant of the nanocomposites was indirectly determined by the relationship of capacitance and relative dielectric constant, defined by the following equation:

$$
\varepsilon_{r}=\frac{C t}{\varepsilon_{0} A}
$$

where $C$ is the capacitance $(\mathrm{F})$, measured by inductancecapacitance-resistance (LCR) meter, $t$ is the thickness $(\mathrm{m})$ of the nanocomposites, and $A$ is the surface area $\left(\mathrm{m}^{2}\right)$ of the electrode. For breakdown strength, it can be directly measured according to ASTM standard [22].

To characterize the influence of nanowire volume fraction on relative dielectric constant, dielectric constants of various volume fractions nanocomposites under frequency of $1 \mathrm{kHz}$ are tested and shown in Figure 6. Results demonstrated an increment in the dielectric permittivity of the 


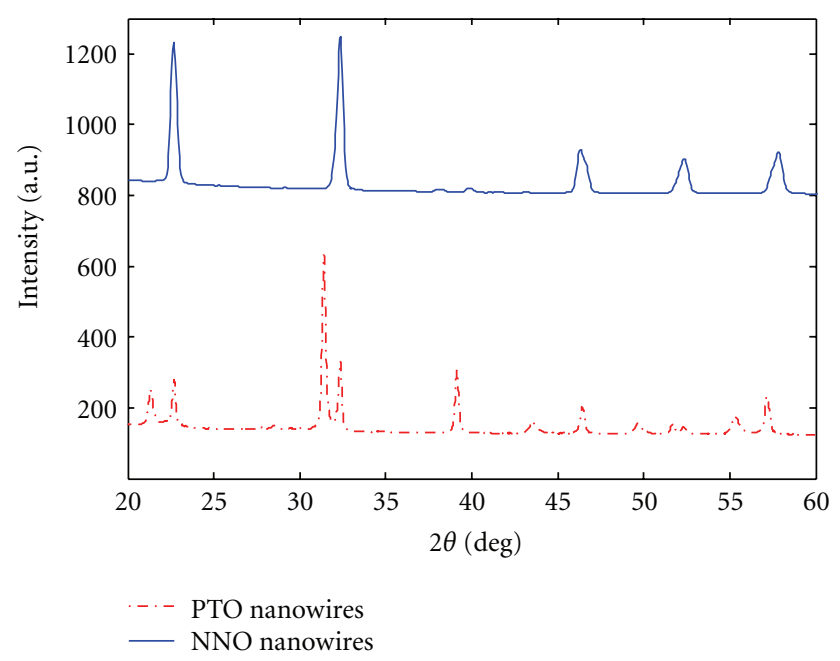

FIgURE 5: XRD patterns of PTO and NNO NWs.

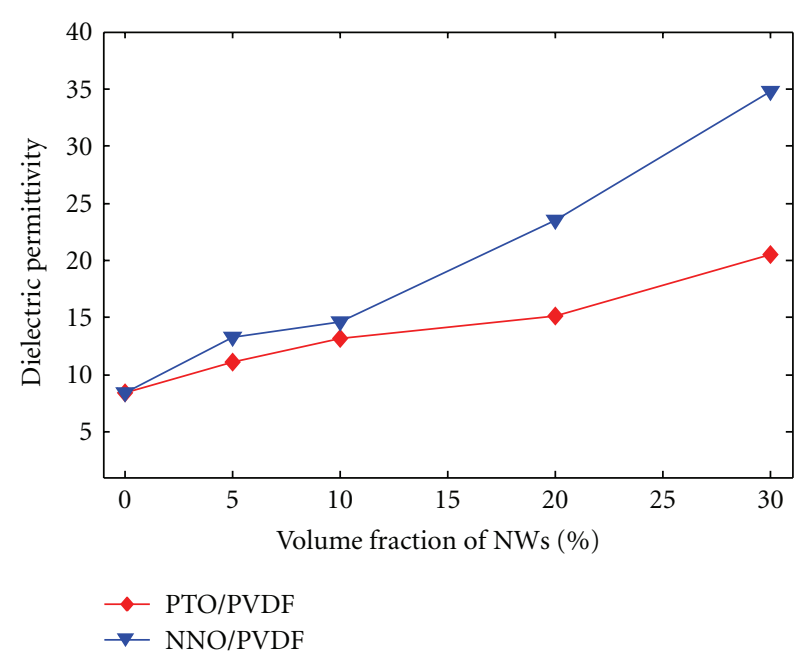

FIGURE 6: Dielectric permittivity constant at $1 \mathrm{kHz}$ of nanocomposites as a function of PTO and NNO NWs volume fractions, (a) NNO NWs/PVDF nanocomposites and (b) PTO NWs/PVDF nanocomposites.

nanocomposites as the volume fraction increased, due to the high dielectric constants of both PTO and NNO, which are about two orders of magnitude higher than that of PVDF matrix $[28,29]$. Note that $0 \%$ volume fraction indicates pure PVDF samples here. Compared with PTO nanocomposites, NNO nanocomposites have shown higher dielectric constant under all volume fractions and frequency ranges. This is because the relative dielectric constant of bulk NNO (209) is higher than that of the PTO $(180)$ at $1 \mathrm{kHz}[30,31]$. For both NNO and PTO nanocomposites, it is shown that the relative dielectric permittivity decreases with the increase of testing frequencies. The average dielectric value exhibited by $30 \%$ $\mathrm{NNO} / \mathrm{PVDF}$ nanocomposites is $34.82,70.1 \%$ higher than that of the $30 \%$ PTO/PVDF nanocomposites.

One of the applications for dielectric capacitor is for high frequency charge and discharge [32]; therefore, the influence of dielectric constant under high frequency must be characterized. The relationship between dielectric permittivity constant and frequency is shown in Figures 7(a) and 7(b). Both PTO and NNO nanocomposites displayed similar behavior under a frequency range between $1 \mathrm{kHz}$ and $1 \mathrm{MHz}$. As frequency increases, dielectric permittivity of the nanocomposites decreases regardless its volume fraction. The general trend demonstrates an exponential decay of dielectric constant; this reduction is more abrupt between $1 \mathrm{kHz}$ and $5 \mathrm{kHz}$. Nanocomposites with $20 \%$ and $30 \%$ volume fraction values displayed a faster reduction in dielectric permittivity when compared with 5\% and 10\% samples. When compared with PTO samples, NNO nanocomposites displayed higher dielectric values among all the frequency ranges tested.

Another important property of capacitors is the dielectric loss, which is a measurement of energy dissipation and degradation of material's insulation [33]. Therefore, the dielectric loss of both NNO and PTO nanocomposites under different volume fractions is measured and shown in Figure 8. It is shown that NNO/PVDF nanocomposites have lower dielectric loss for all volume fraction samples. High volume fraction nanocomposites exhibited lower loss because of the lower dielectric loss of the ceramics, compared with polymer matrix.

After the characterization of dielectric constant and loss of the nanocomposites, breakdown strength testing is carried out to fully understand the energy density. Figure 9 shows the breakdown strength of both NNO/PVDF and PTO/PVDF nanocomposites. Because the dielectric strength of PVDF is orders of magnitude higher than the nanowire inclusions, the dielectric strength decreases with the increase of volume fraction for both types of nanocomposites. Pure PVDF matrix has a breakdown strength of $299 \mathrm{MV} / \mathrm{m}$. Starting from a volume fraction of $20 \%$ PTO and NNO NWs, the breakdown field starts to stabilize and decreases at a slower rate; this behavior is similar to previous research efforts [19]. The breakdown strength of $30 \%$ PTO/PVDF nanocomposites doubles the value recorded for 30\% NNO/PVDF nanocomposites, 159.647 to $63.463 \mathrm{KV} / \mathrm{m}$.

The maximum energy density calculated from the experimental dielectric constant and dielectric strength is shown in Figure 10(a). Breakdown strength plays a more important role than dielectric constant (square versus linear) in governing the energy density, as indicated by (1). Although the dielectric constant of NNO/PVDF is higher, its dielectric breakdown strength is lower, leading to lower energy density, as shown in Figure 10(a). Note that Figure 10(a) shows the volume energy density $\left(\mathrm{J} / \mathrm{cm}^{3}\right)$ and Figure $10(\mathrm{~b})$ shows the mass energy density $(\mathrm{J} / \mathrm{g})$; dielectric constants used are based on $1 \mathrm{kHz}$ testing frequency $[19,34]$.

The trend for energy densities for both NNO/PVDF and PTO/PVDF decreases first then increases with the increase of volume fraction, with the only exception of $5 \%$ PTO/PVDF as shown in Figure 10(a). This phenomenon is caused by the combined influence of dielectric constant and breakdown strength on energy density. As shown in (1), the energy density is governed by both dielectric constant and breakdown strength. With the increase of nanowire volume 


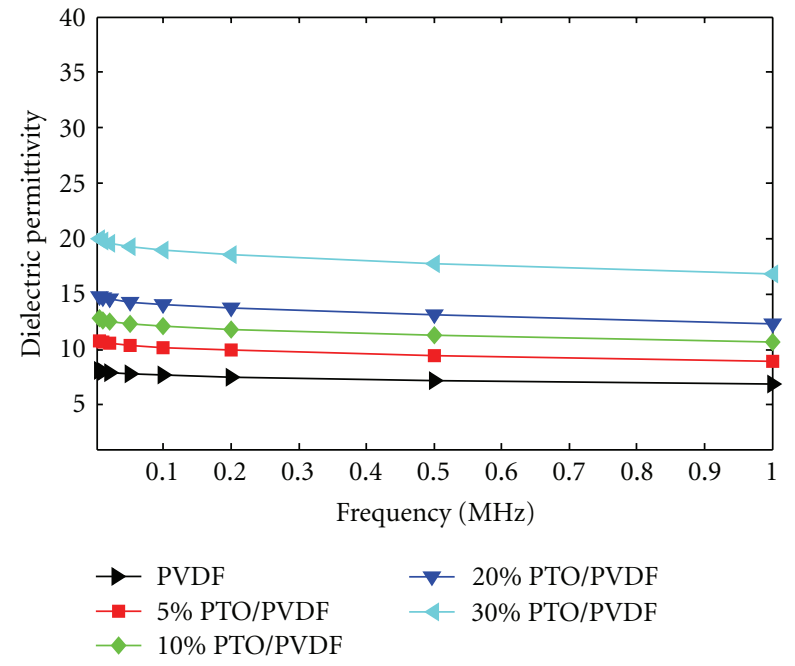

(a)

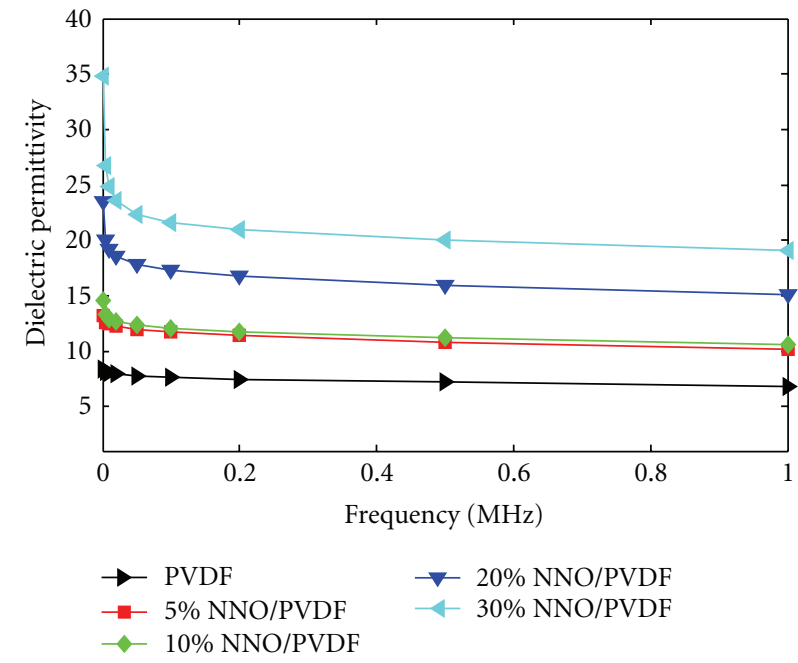

(b)

Figure 7: Dielectric permittivity constant of PTO and NNO nanocomposites between a frequency range of $1 \mathrm{kHz}-1 \mathrm{MHz}$, (a) PTO NWs/PVDF nanocomposites and (b) NNO NWs/PVDF nanocomposites.

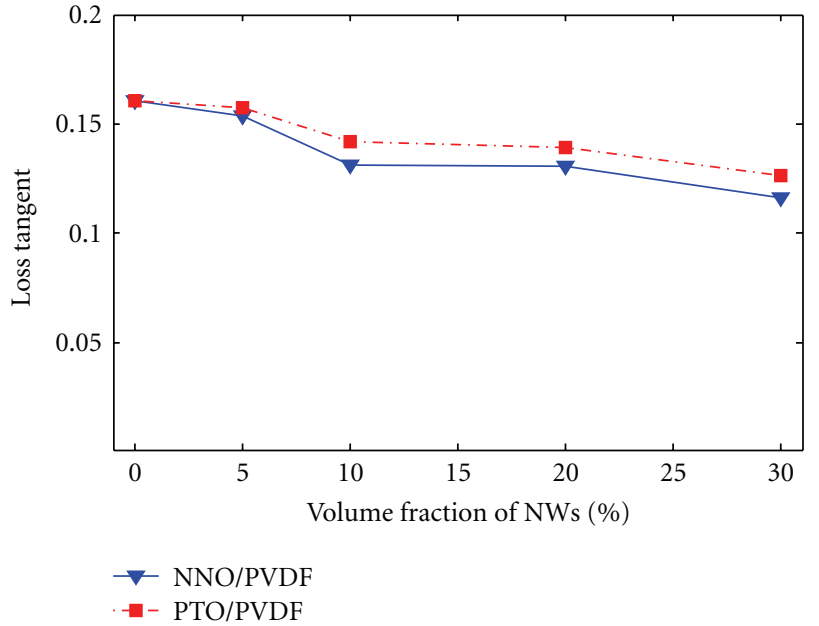

Figure 8: Dielectric loss tangent at $1 \mathrm{MHz}$ of nanocomposites as a function of PTO and NNO NWs volume fraction.

fraction, the dielectric constant increases while breakdown strength decreases; at low volume fraction, the effect of decrease in breakdown strength is dominant while at high volume fraction, the effect of increase in dielectric constant is more significant. For mass energy density of 5\% PTO/PVDF, the breakdown strength did not decrease much, thus leading to an increase in mass energy density.

Because weight is equally important as volume in modern materials system design, the mass energy density is also plotted and shown in Figure 10(b). NNO/PVDF nanocomposites have lower volume and mass energy density than PTO/PVDF system throughout the entire volume fraction range, with a minimum difference of $45.5 \%$. Compared with previously developed PZT/PVDF nanocomposites, NNO/PVDF displayed similar energy density. Between

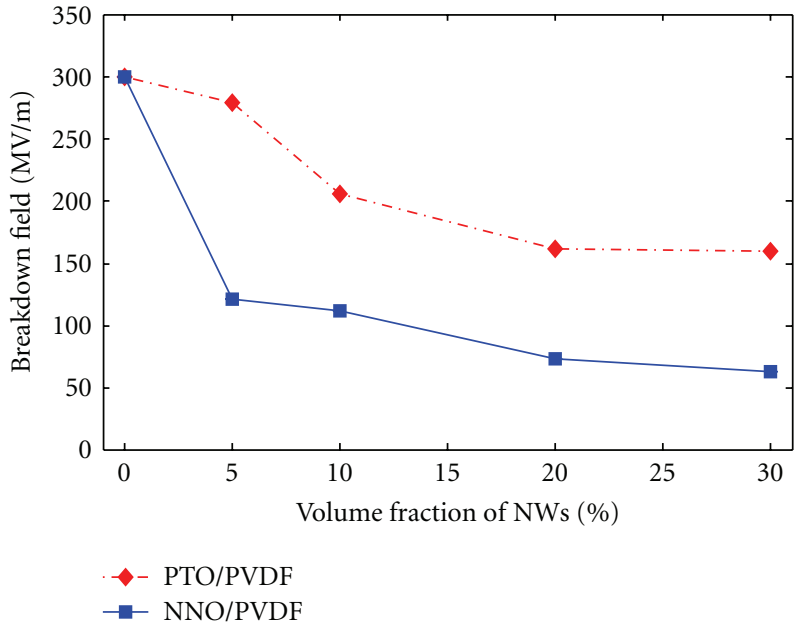

FIGURE 9: Breakdown strength of PTO and NNO nanocomposites as a function of volume fraction of NWs.

the volume fraction range of $20 \%$ and $30 \%$, the minimum and maximum differences in terms of energy density between PZT/PVDF and NNO/PVDF randomly oriented nanocomposites are only 5.82 and $12.9 \%$, respectively [19, 34]. Besides, available methods such as nanowire surface functionalization [34] and nanowire alignment [18] could lead to further increase of energy density of the NNO/PVDF nanocomposites, leading to lead-free energy storage devices for the next generation biologically safe and environmentally benign materials systems.

\section{Conclusions}

This paper introduces the fabrication and characterization of a lead-free nanocomposites dielectric capacitor based 


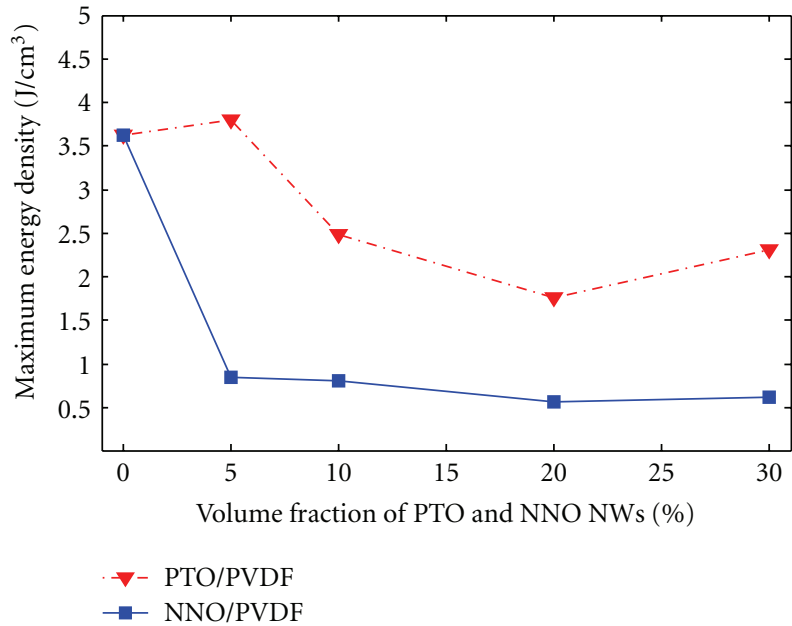

(a)

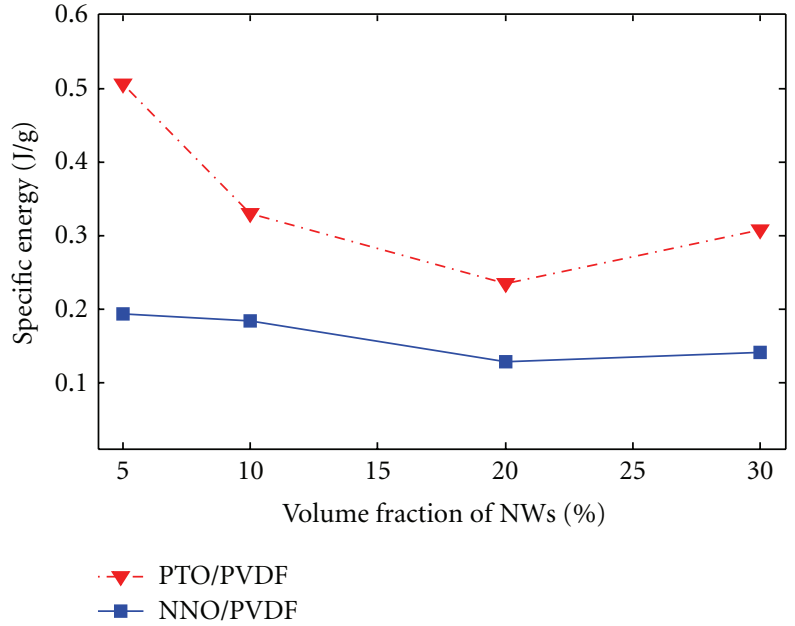

(b)

FIGURE 10: Energy density of the nanocomposites, (a) calculated maximum energy density of nanocomposites with different volume fractions of PTO and NNO nanowires and (b) specific energy of PTO and NNO nanocomposites as a function of volume fraction.

on $\mathrm{NaNbO}_{3}$ nanowires embedded in PVDF matrix. It is shown that the $\mathrm{NaNbO}_{3} / \mathrm{PVDF}$ nanocomposites have higher dielectric constants than $\mathrm{PbTiO}_{3} / \mathrm{PVDF}$ composites, with largest difference of $70.1 \%$. Also, the dielectric loss of $\mathrm{NaNbO}_{3} / \mathrm{PVDF}$ composites is lower than that of the $\mathrm{PbTiO}_{3} / \mathrm{PVDF}$ composites. The energy density of various volume fraction $\mathrm{NaNbO}_{3} / \mathrm{PVDF}$ nanocomposites ranges from $0.5675 \mathrm{~J} / \mathrm{cm}^{3}$ to $0.8511 \mathrm{~J} / \mathrm{cm}^{3}$ or $0.1404 \mathrm{~J} / \mathrm{g}$ to $0.1925 \mathrm{~J} / \mathrm{g}$. Comparing with $\mathrm{PbTiO}_{3} / \mathrm{PVDF}$ or $\mathrm{PbZrTiO}_{3} / \mathrm{PVDF}$ nanocomposites, the energy densities of $\mathrm{NaNbO}_{3} / \mathrm{PVDF}$ nanocomposites are lower but comparable, with a minimal difference of $5.82 \%$. Further surface functionalization and inclusion alignment could significantly enhance the energy density of the lead-free nanocomposite capacitors. Therefore, this research effort has shown the feasibility of expelling lead from high dielectric constant and high-energy density capacitors in the future.

\section{References}

[1] P. Barber, S. Balasubramanian, Y. Anguchamy et al., "Polymer composite and nanocomposite dielectric materials for pulse power energy storage," Materials, vol. 2, pp. 1697-1733, 2009.

[2] P. J. Grbović, P. Delarue, P. Le Moigne, and P. Bartholomeus, "The ultracapacitor-based controlled electric drives with braking and ride-through capability: overview and analysis," IEEE Transactions on Industrial Electronics, vol. 58, no. 3, pp. 925-936, 2011.

[3] Y. Zhang, H. Feng, X. Wu et al., "Progress of electrochemical capacitor electrode materials: a review," International Journal of Hydrogen Energy, vol. 34, no. 11, pp. 4889-4899, 2009.

[4] Y. Rao, S. Ogitani, P. Kohl, and C. P. Wong, "Novel polymerceramic nanocomposite based on high dielectric constant epoxy formula for embedded capacitor application," Journal of Applied Polymer Science, vol. 83, no. 5, pp. 1084-1090, 2002.

[5] P. Lehmann, "Overview of the electric launch activities at the French-German research Institute of Saint-Louis (ISL)," IEEE Transactions on Magnetics, vol. 39, no. 1, pp. 24-28, 2003.
[6] T. Tanaka, G. C. Montanari, and R. Mülhaupt, "Polymer nanocomposites as dielectrics and electrical insulationperspectives for processing technologies, material characterization and future applications," IEEE Transactions on Dielectrics and Electrical Insulation, vol. 11, no. 5, pp. 763-784, 2004.

[7] Z. Tian, X. Wang, L. Shu et al., "Preparation of nano $\mathrm{BaTiO}_{3}$ based ceramics for multilayer ceramic capacitor application by chemical coating method," Journal of the American Ceramic Society, vol. 92, no. 4, pp. 830-833, 2009.

[8] M. Unruan, T. Sareein, J. Tangsritrakul et al., "Changes in dielectric and ferroelectric properties of $\mathrm{Fe}^{3+} / \mathrm{Nb}^{5+}$ hybriddoped barium titanate ceramics under compressive stress," Journal of Applied Physics, vol. 104, no. 12, Article ID 124102, 2008.

[9] O. Guillon, J. Chang, S. Schaab, and S.-J. L. Kang, "Capacitance enhancement of doped barium titanate dielectrics and multilayer ceramic capacitors by a post-sintering thermomechanical treatment," Journal of the American Ceramic Society, vol. 95, no. 7, pp. 2277-2281, 2012.

[10] S. S. Ibrahim, A. A. Al Jaafari, and A. S. Ayesh, "Physical characterizations of three phase polycarbonate nanocomposites," Journal of Plastic Film and Sheeting, vol. 27, no. 4, pp. 275-291, 2011.

[11] L. Xie, X. Huang, C. Wu, and P. Jiang, "Core-shell structured poly(methyl methacrylate) $/ \mathrm{BaTiO}_{3}$ nanocomposites prepared by in situ atom transfer radical polymerization: a route to high dielectric constant materials with the inherent low loss of the base polymer," Journal of Materials Chemistry, vol. 21, no. 16, pp. 5897-5906, 2011.

[12] P. Kim, N. M. Doss, J. P. Tillotson et al., "High energy density nanocomposites based on surface-modified $\mathrm{BaTiO}_{3}$ and a ferroelectric polymer," ACS Nano, vol. 3, no. 9, pp. 2581-2592, 2009.

[13] Y. P. Mao, S. Y. Mao, Z.-G. Ye, Z. X. Xie, and L. S. Zheng, "Sizedependences of the dielectric and ferroelectric properties of $\mathrm{BaTiO}_{3} /$ polyvinylidene fluoride nanocomposites," Journal of Applied Physics, vol. 108, no. 1, Article ID 014102, 2010.

[14] Z.-M. Dang, J.-K. Yuan, J.-W. Zha, T. Zhou, S.-T. Li, and G.-H. Hu, "Fundamentals, processes and applications of 
high-permittivity polymer-matrix composites," Progress in Materials Science, vol. 57, no. 4, pp. 660-723, 2012.

[15] L. Ni and X. M. Chen, "Dielectric relaxations and formation mechanism of giant dielectric constant step in $\mathrm{CaCu}_{3} \mathrm{Ti}_{4} \mathrm{O}_{12}$ ceramics," Applied Physics Letters, vol. 91, no. 12, Article ID 122905, 2007.

[16] A. Chen, K. Kamata, M. Nakagawa, T. Iyoda, H. Wang, and X. $\mathrm{Li}$, "Formation process of silver-polypyrrole coaxial nanocables synthesized by redox reaction between $\mathrm{AgNO}_{3}$ and pyrrole in the presence of poly(vinylpyrrolidone)," Journal of Physical Chemistry B, vol. 109, no. 39, pp. 18283-18288, 2005.

[17] D. K. Das-Gupta and K. Doughty, "Polymer-ceramic composite materials with high dielectric constants," Thin Solid Films, vol. 158, no. 1, pp. 93-105, 1988.

[18] C. Andrews, Y. Lin, and H. A. Sodano, "The effect of particle aspect ratio on the electroelastic properties of piezoelectric nanocomposites," Smart Materials and Structures, vol. 19, no. 2, Article ID 025018, 2010.

[19] H. Tang, Y. Lin, C. Andrews, and H. A. Sodano, "Nanocomposites with increased energy density through high aspect ratio PZT nanowires," Nanotechnology, vol. 22, no. 1, Article ID 015702, 2011.

[20] J. H. Jung, M. Lee, J. I. Hong et al., "Lead-free $\mathrm{NaNbO}_{3}$ nanowires for high output piezoelectric nanogenerator," ACS Nano, vol. 5, Article ID 10041, 2011.

[21] J. Wang, C. S. Sandu, and N. Setter, "Large-scale fabrication of titanium-rich perovskite PZT submicro/nano wires and their electromechanical properties," IEEE Transactions on Ultrasonics, Ferroelectrics, and Frequency Control, vol. 56, no. 9, pp. 1813-1819, 2009.

[22] ASTM Standard D149-09, "Standard test method for dielectric breakdown voltage and dielectric strength of solid electrical insulating materials at commercial power frequencies," Research Report, ASTM International, West Conshohocken, Pa, USA, 2009.

[23] H. Shi, X. Li, D. Wang, Y. Yuan, Z. Zou, and J. Ye, " $\mathrm{NaNbO}_{3}$ nanostructures: facile synthesis, characterization, and their photocatalytic properties," Catalysis Letters, vol. 132, no. 1-2, pp. 205-212, 2009.

[24] T. Y. Ke, H. A. Chen, H. S. Sheu et al., "Sodium niobate nanowire and its piezoelectricity," Journal of Physical Chemistry $C$, vol. 112, no. 24, pp. 8827-8831, 2008.

[25] X. Lu, D. Zhang, Q. Zhao, C. Wang, W. Zhang, and Y. Wei, "Large-scale synthesis of necklace-like single-crystalline $\mathrm{PbTiO}_{3}$ nanowires," Macromolecular Rapid Communications, vol. 27, no. 1, pp. 76-80, 2006.

[26] Z. Cai, X. Xing, R. Yu, X. Sun, and G. Liu, "Morphologycontrolled synthesis of lead titanate powders," Inorganic Chemistry, vol. 46, pp. 7423-7427, 2007.

[27] B. Chu, X. Zhou, K. Ren et al., "A dielectric polymer with high electric energy density and fast discharge speed," Science, vol. 313, no. 5785, pp. 334-336, 2006.

[28] V. V. Varadan, Y. R. Roh, V. K. Varadan, and R. H. Tancrell, "Measurement of all the elastic and dielectric constants of poled PVDF films," in Proceedings of the IEEE Ultrasonics Symposium, vol. 2, pp. 727-730, October 1989.

[29] Y. I. Yuzyuk, P. Simon, E. Gagarina et al., "Modulated phases in $\mathrm{NaNbO}_{3}$ : Raman scattering, synchrotron $\mathrm{x}$-ray diffraction, and dielectric investigations," Journal of Physics Condensed Matter, vol. 17, no. 33, pp. 4977-4990, 2005.

[30] V. Shanker, S. L. Samal, G. K. Pradhan, C. Narayana, and A. K. Ganguli, "Nanocrystalline $\mathrm{NaNbO}_{3}$ and $\mathrm{NaTaO}_{3}$ : rietveld studies, Raman spectroscopy and dielectric properties," Solid State Sciences, vol. 11, no. 2, pp. 562-569, 2009.

[31] Y. Hu, H. Gu, W. Chen, and Y. Wang, "Preparation of $\mathrm{PbTiO}_{3}$ nanoceramics based on hydrothermal nanopowders and characterization of their electrical properties," Materials Chemistry and Physics, vol. 121, no. 1-2, pp. 10-13, 2010.

[32] K. M. Slenes, P. Winsor, T. Scholz, and M. Hudis, "Pulse power capability of high energy density capacitors based on a new dielectric material," IEEE Transactions on Magnetics, vol. 37, no. 1, pp. 324-327, 2001.

[33] Q. Li, T. Zhao, and W. H. Siew, "Definition and digital algorithms of dielectric loss factor for condition monitoring of high-voltage power equipment with harmonics emphasis," IEE Proceedings Generation, Transmission and Distribution, vol. 152, no. 3, pp. 309-312, 2005.

[34] H. Tang, Y. Lin, and H. A. Sodano, "Enhanced energy storage in nanocomposite capacitors through aligned PZT nanowires by uniaxial strain assembly," Advanced Energy Materials, vol. 2, no. 4, pp. 469-476, 2012. 

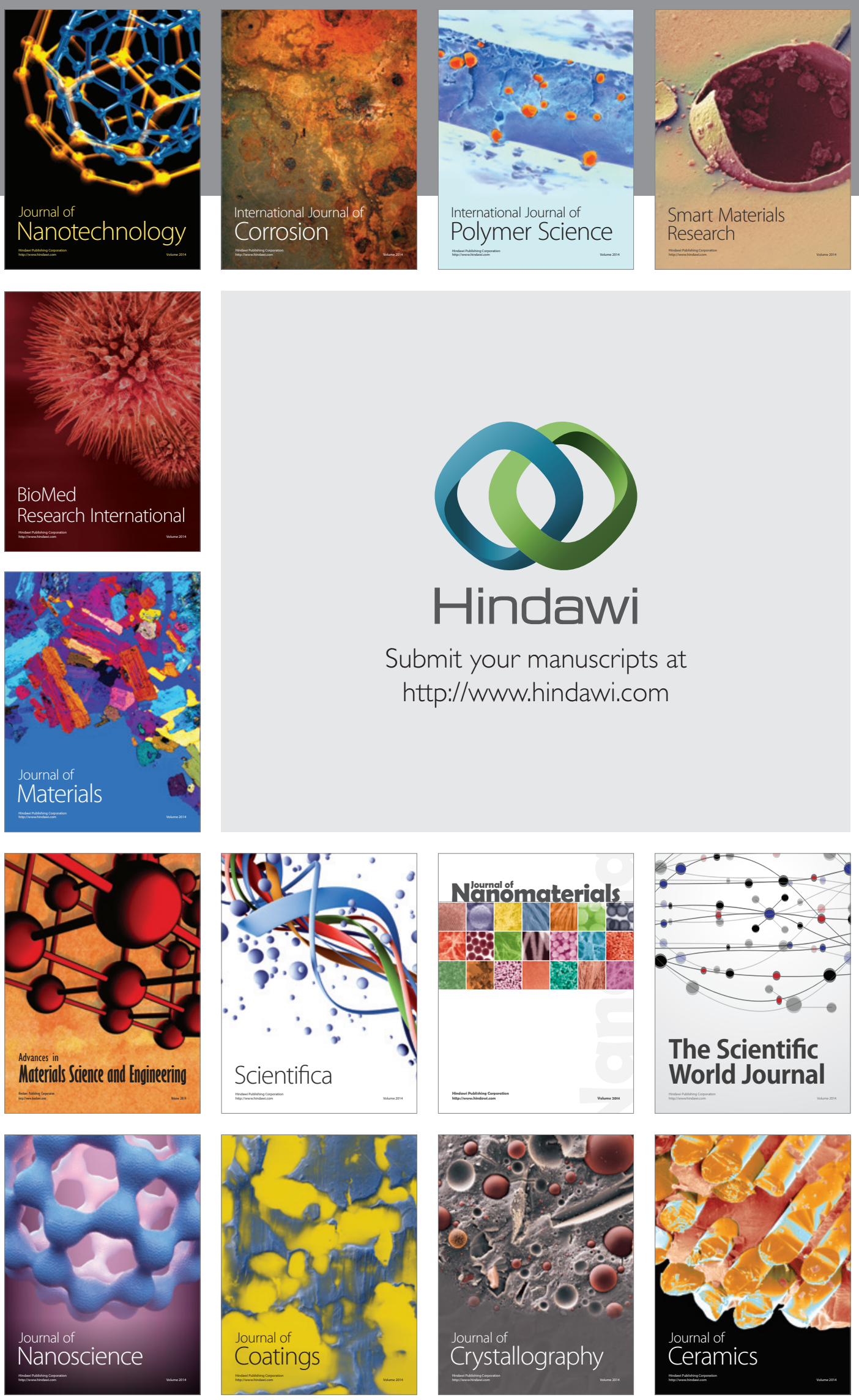

The Scientific World Journal

Submit your manuscripts at

http://www.hindawi.com

\section{World Journal}

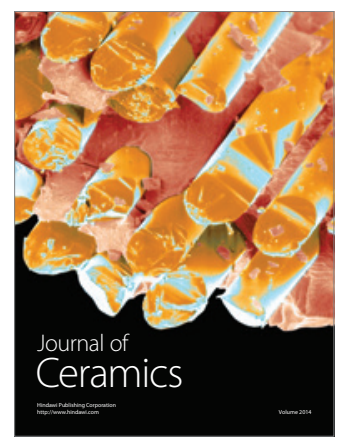

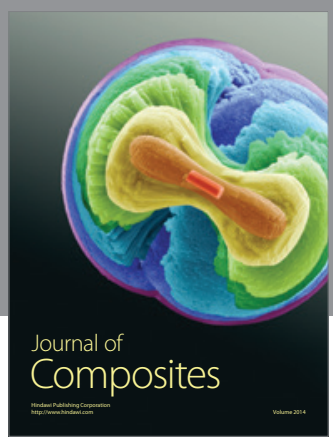
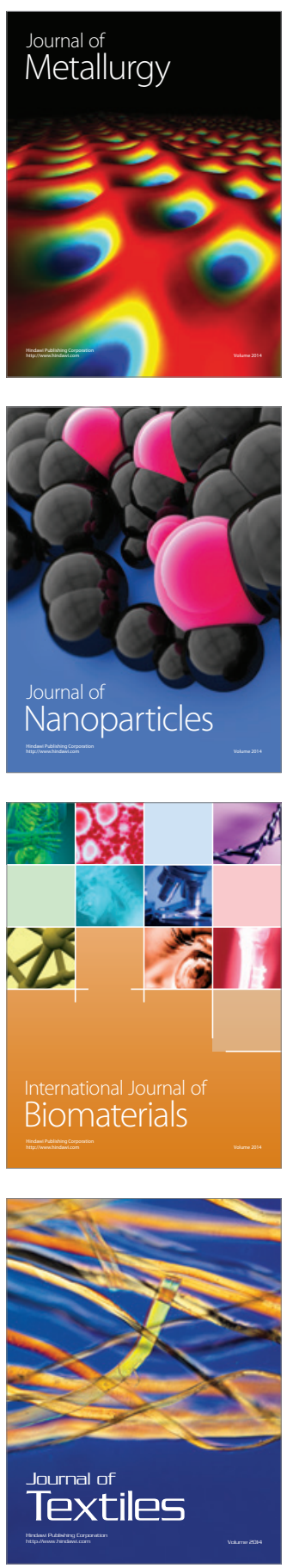